\title{
COMPTE-RENDUS DE CONGRÈS \\ ET REVUE DE LA LITTÉRATURE INTERNATIONALE
}

Avec la collaboration de :

J.C.CZYBA

M.DROSDOWSKY

P.FENICHEL

H.LEJEUNE

N.MOTTET

J.PARINAUD

E.SZERMAN-JOLY 


\section{Compte-rendus de Congrès \\ et Revue de la littérature internationale}

\section{SPERMIOLOGIE-SPERMATOGENÈSE}

109 Compte-rendu du colloque international sur la réaction acrosomique du spermatozoïde humain

Collioure, 7-9 septembre 1995 J.PARINAUD et P.FENICHEL

113 Compte-rendu du 15ème Congrès mondial sur la fertilité et la stérilité (IFFS) Montpellier, 17-22 septembre 1995 E.SZERMAN-JOLY

118 Différents types d'altération de la spermatogenèse provoqués par des délétions du chromosome $Y$ emportant le gène d'une nouvelle protéine de liaison de l'ARN.

R.REIJO, TY LEE, P.SALO et al Nature Genetics, 1995, $10: 383-393$

119 Oscillations du calcium dans les œufs de mammifères déclenchée par une protéine soluble des spermatozoïdes.

J.PARRINGTON, K.SWANN, V.I.SCHECHENKO et al. Nature, 1995, 379 : 364-368.

\section{ENDOCRINOLOGIE}

120 Analyse quantitative du messager du récepteur des androgènes dans les cellules de Leydig et les cellules de Sertoli en cours de développement par hybridation in situ.

LI-XIN SHAN, LI-JI-ZHU, C.W.BARDIN, M.P.HARDY.

Endocrinology, 1995, $136: 3856-3862$.

121 Etude prospective des profils hormonaux et séminaux chez des coureurs de marathon.

C.E.JENSEN, K.WISWEDEL, J.Mc LOUGHLIN, Z.van der SPUY Fertility and Sterility, 1995, $64: 1189-1196$

\section{IMMUNOLOGIE}

122 Mécanisme des rejets de greffe.

D.L. VAUX Nature, 1995,377 : 576-577.

\section{CANCER}

123 Cancer et stérilité masculine

D.MEIROW, J.G.SCHENKER Human Reproduction, 1995, 10 : 2017-2022

\section{FÉCONDATION IN VITRO}

125 Développement de souris normales à partir d'ovocytes injectés avec un noyau de spermatocyte secondaire YASUYURI KIMURA, R.YAGANIMACHI

Biology of Reproduction, 1995, $53: 855-862$. 


\section{SPERMIOLOGIE - SPERMATOGENÈSE}

\section{Compte-rendu du colloque international}

\section{LA RÉACTION ACROSOMIQUE DU SPERMATOZOIDE HUMAIN}

\author{
J.PARINAUd, P.FEnICHEL \\ Collioure, 7-9 septembre 1995
}

\section{Introduction}

Le symposium a réuni à Collioure 127 participants provenant de 22 pays différents. L'ensemble des communications a été effectué par les plus grands spécialistes mondiaux dans le domaine et tous les orateurs prévus sur le programme étaient présents ce qui fait de ce symposium un des plus beaux plateaux scientifiques jamais réuni dans l'infertilité masculine.

De plus les participants étaient tous des spécialistes travaillant depuis de nombreuses années sur ce sujet, permettant ainsi de donner tout leur intérêt aux larges plages de discussion aménagées à cet effet.

La session poster a présenté 47 posters répartis selon les 4 thèmes clefs du colloque (capacitation, induction de la réaction acrosomique, transduction du signal et applications cliniques).

Un résumé des différentes sessions est présenté ci-dessous. L'intégralite des communications invités et les résumés des posters sont publiés dans une co-édition INSERM/John LibBey Eurotext. Ce livre "Réaction Acrosomique du Spermatozoüde humain " P. FÉNICHEL ET J. PARINAUD éditeurs, peut être obtenu auprès de John Libbey Eurotext, 127 rue de la république, 92120 Montrouge.

\section{Capacitation}

Conçu il y a plus de 50 ans comme l'acquisition de la fécondance du sperme dans les voies génitales femelles, ce processus, sans substratum morphologique, est resté longtemps sans explication. Il peut aujourd'hui être rapporté à des changements moléculaires touchant la membrane plasmique du spermatozoïde, permettant ainsi la liaison à la zone pellucide et l'induction de la réaction acrosomique (RA).

William Holt (Londres, Grande Bretagne) a en effet illustré, à l'aide d'analyses moléculaires récentes des interactions lipides/protéines spécifiques, l'architecture complexe et différenciée dans le temps et dans l'espace de la membrane plasmique du spermatozoïde humain. Elle est organisée en domaines et subit d'importantes modifications depuis la spermatogénèse jusqu'à la fécondation. Au cours de la capacitation la membrane se prépare à la transduction du signal et à la fusion qui caractérise la RA.

Robin Harrison (Cambridge, Grande Bretagne) a précisé, à l'aide d'une sonde lipophile et de phospholipides marqués, les changements de l'architecture lipidique de la membrane plasmique, induits par les bicarbonates. Ces changements, liés à des mécanismes enzymatiques, favorisent la perte des molécules de cholestérol, étape importante de la capacitation grâce à des facteurs d'épuration présents dans les fluides génitaux et dans les milieux de culture appropriés. Charles Muller (Seattle, USA) a isolé et caractérisé une protéine de transfert lipidique, LTP-1, à partir du liquide folliculaire. Cette protéine de type HDL contamine la plupart des préparations commerciales d'albumine et participe très vraisemblablement à l'induction de la capacitation en abaissant le rapport cholestérol/phospholipides. La fluidification de la membrane induite par ces modifications lipidiques conduit à une relocalisation par migration des protéines et entraîne ainsi une série d'événements membranaires et cytoplasmiques (mouvements ioniques, phosphorylation de protéines sur tyrosine...) indispensables à la survenue de la RA. 
Lynn Fraser (Londres, Grande Bretagne) a précisé ces flux ioniques surtout sur la tête du spermatozoïde. Ils impliquent au cours de la capacitation, chez la souris,

1. un influx de calcium lent et modéré par inhibition d'une $\mathrm{Ca}^{2+}$ ATPase, différent du flux massif et rapide via des canaux calciques de la RA,

2. un influx de Na présent en grande quantité dans les fluides génitaux,

3 . des bicarbonates qui pourraient avoir une double action (contrôle de $\mathrm{pH}$ et stimulation de l'adénylate cyclase) et enfin

\section{4. l'intervention de l'ion chlorure.}

HARRY MooRe (Sheffield, Grande Bretagne) a expliqué qu'un état de capacitation complet exigeait la mise en place de voies de transduction d'une ou de plusieurs tyrosine kinases en particulier d'une protéine de 95 $\mathrm{kD}$ (récepteur présumé de ZP3) dont la phosphorylation progressive, jusqu'à un certain seuil, conduisait après agrégation à la RA soit spontanément soit par liaison du ligand naturel ZP3.

\section{Induction de la réaction acrosomique}

Dans la 2ème session consacrée à l'induction physiologique de la RA, PATricia SALING (Durham, USA) a montré que cette protéine de $95 \mathrm{kD}$ appelée ZRK (zona receptor kinase) a été identifiée, clonée et séquencée pour le spermatozoïde humain à partir de ZRK murin. Elle possède des caractéristiques de la famille des tyrosine kinases et participe à la liaison à ZP3 et à l'induction de l'exocytose acrosomique. Christopher Barratt (Sheffield, Grande Bretagne) a réussi à produire un recombinant de ZP3 humain (rhuZP3) à partir de cellules ovariennes de hamster transfectées. La rhuZP3 est capable d'induire un taux élevé de RA sur des spermatozoïdes humains même peu capacités; elle constitue un outil privilégié pour préciser les caractéristiques biochimiques de la liaison ZP3/rZP3 et pour proposer après purifica- tion un inducteur physiologique dans l'exploration clinique des troubles de la RA. Jan Tesarik (Paris, France) a évoqué le rôle possible des protéases (acrosine et acrosine like) au cours de la RA qui est à la fois une activation cellulaire médiée par des récepteurs et une fusion cellulaire. Patricio Morales (Santiago, Chili) a passé en revue les protéines impliquées dans le franchissement par le spermatozoïde des enveloppes de l'oeuf, y compris la membrane ovocytaire en rapportant en particulier le rôle probable d'une activité trypsine-like associée à la membrane du spermatozoïde dans l'induction de la RA par la ZP.

STANLey Meizel (Davis, USA) a fait le point sur l'effet non génomique de la progestérone sur le spermatozoïde humain capable d'induire un influx calcique, un efflux de chlore et la RA. Il a décrit sur le spermatozoïde un récepteur apparenté au récepteur $\mathrm{GABA}_{\mathrm{A}} /$ canal Chlore et soulevé l'hypothèse d'un second récepteur médiant l'influx calcique. La question du rôle physiologique de la progestérone dans l'induction de la RA en potentialisant l'action de ZP3 a également été discutée. Peter Blackmore (Norfolk, USA) a de son côté tenté d'approcher les caractéristiques du récepteur membranaire à la progestérone, responsable dans le spermatozoïde humain de l'influx de $\mathrm{Ca}^{2+}$, en utilisant 160 analogues. Ces résultats évoquent un récepteur différent du canal $\mathrm{GABA}_{\mathrm{A}} /$ canal Chlore, un rôle important joué par le méthyl en C21 et un site de liaison pour le récepteur spermatique sur la face $b$ des noyaux $C$ et $D$ du stéroïde contrairement à la liaison au récepteur génomique.

\section{Les voies de transduction du signal au cours de la réaction acrosomique}

Cette session a été consacrée aux mécanismes mis en jeu après la liaison des inducteurs de la réaction acrosomique (zone pellucide, progestérone) à leurs récepteurs membranaires. 
HARVEy Florman (Shrewsbury, USA) a décrit un modèle d'influx de calcium induit par ZP3. Lors de la liaison de ZP3 à son récepteur, il y a d'une part ouverture d'un canal cationique peu sélectif entraînant une dépolarisation de la membrane et d'autre part activation d'une $G$ protéine agissant sur un régulateur de pHi augmentant celuici. Ces deux réponses permettent l'ouverture des canaux calciques conduisant à la réaction acrosomique.

Gregory KOPF (Philadelphia, USA) a rappelé que le spermatozoïde humain ne contenait que des protéines Gi et pas de Gs. Les protéines Gi sont stimulées par ZP3 (uniquement issue d'ovocytes non fécondés) et modulent l'ouverture des canaux calciques. La ZP3 active également l'adenyl cyclase entraînant une augmentation de l'AMPc et l'activation d'une protéine kinase A. CHRISTOPHER DE JONGE (Omaha, USA) a montré que les analogues de l'AMPc et les activateurs de la PKA induisait la réaction acrosomique.

Ebouardo Roldan (Madrid, Espagne) a montré que la stimulation de la réaction acrosomique par la zone pellucide, la progestérone ou le calcium ionophore entraîne l'hydrolyse du phosphatidyl inositol par stimulation d'une phospholipase $\mathrm{C}$. Ceci permet la formation de diacylglycerols qui stimulent d'une part une phospholipase A2, qui va augmenter le taux de DAG, et d'autre part une protéine kinase C. ZVI NAOR (Tel Aviv, Israel) a rapporté que la PKC était localisée essentiellement au niveau du segment équatorial, mais aussi au niveau du flagelle. La stimulation de la PKC par les esters de phorbol entraîne une augmentation calcium dépendante de la réaction acrosomique et de la mobilité.

C. DE JoNGE a décrit les interconnections très étroites entre la voie de la PKA et celle de la PKC. Leurs activations simultanées ont un effet additif sur la réaction acrosomique, alors que toute inhibition d'une voie diminue les effets de l'autre.
RAJESH NAZ (New York, USA) a montré que la capacitation et la réaction acrosomique augmentent le degré de phosphorylation des spermatozoïdes. Deux protéines, $95 \mathrm{kD}$ et $51 \mathrm{kD}$ (antigène $\mathrm{FA1}$ ), sont phosphorylées sur tyrosine et interviennent dans la liaison du spermatozoïde sur la zone pellucide.

Au total, comme rapporté par G. KoPF, il apparaît que l'attachement du spermatozoïde sur la zone pellucide met en jeu de multiples récepteurs impliquant des voies de transmission du signal différentes mais interconnectées.

\section{Applications cliniques}

Cette session a été consacrée aux méthodes d'évaluation de la fonction acrosomique, à l'origine et aux conséquences des dysfonctionnements de l'acrosome ainsi qu'à la modulation in vivo et vitro de la réaction acrosomique.

L'étude de la fonction acrosomique se heurte à deux problèmes: le choix

1. de la technique de mise en évidence et

2.de l'inducteur de la réaction acrosomique utilisé pour les tests dynamiques. Nicholas CRoss (Stillwater, USA) a présenté les différentes techniques utilisées et a souligné la difficulté de leur choix, certaines permettant de visualiser les tout premiers stades de la réaction acrosomique, d'autres ne mettant en évidence que les réactions acrosomiques complètes. Selon lui, la technique idéale, qui malheureusement n'existe pas, doit permettre d'identifier les premiers stades de la réaction acrosomique, être utilisable en microscopie à fond clair ou en cytométrie de flux et ne pas altérer les fonctions spermatiques de façon à pouvoir associer l'étude de la mobilité à celle de la réaction acrosomique. DAvid MorTimer (Sydney, Australie) a rapporté que l'inducteur idéal serait la protéine ZP3 de la zone pellucide, mais les faibles quantités disponibles interdisent son utilisation en routine et la ZP3 
recombinante n'est pas encore disponible. Parmi les autres inducteurs, le liquide folliculaire pose des problèmes de standardisation et seuls la progestérone et le ionophore A23187 sont réellement utilisables. D Mortimer préconise l'utilisation de ce dernier en utilisant de faibles concentrations. Une standardisation des protocoles d'évaluation de la fonction acrosomique semble indispensable. PATRICK FÉnICHEL (Nice, France) a montré que la réaction acrosomique spontanée était plus élevée et la réponse au ionophore A23187 plus faible dans les échecs de fécondation in vitro que dans les succès. Cette approche clinique a permis d'identifier des déficits spermatiques non accessibles par les moyens classiques et d'expliquer certains échecs de fécondation in vitro malgré un spermogramme normal. FRANK KoHN (Giessen, Allemagne) a rapporté que la présence de varicocèle ou des facteurs environnementaux, tels que la consommation tabagique ou l'exposition au chlorure de platine, entraînent des dysfonctions acrosomiques qui apparaissent avant toutes anomalies du spermogramme. JOHN AITKEN (Edinburgh, Ecosse) a révélé que l'excès de production d'anions superoxides par les leucocytes du sperme peut engendrer une peroxidation des lipides membranaires, altérant l'intégrité des membranes du spermatozoïde et donc la fonction acrosomique. Par contre, ces mêmes radicaux libres produits en faible quantité par les spermatozoïdes sont indispensables à la capacitation et à la réaction acrosomique.

La fonction acrosomique peut être modulée in vitro afin d'augmenter les chances de succès de la fécondation in vitro. Ainsi, Yves MÉNÉzo (Villeurbanne, France) a rapporté que la composition ionique du milieu peut modifier la capacitation et que le bicarbonate augmente le taux d'AMPc et donc la capacitation. L'utlisation d'agents antioxydants (hypotaurine, $\mathrm{N}$-acetylcystéine, glutathion) peut permettre de limiter l'action délétère des radicaux libres et de maintenir les fonctions spermatiques (Y. MÉNÉzo ET J.
AitKen). Enfin, D. MorTimer et P. FÉniCHeL ont montré que l'on pouvait augmenter in vitro la capacité des spermatozoïdes à réaliser la réaction acrosomique en utilisant des agents pharmacologiques tels que la pentoxifylline ou le NECA.

Les techniques de micro-injections se sont grandement développées ces dernières années. ANDRE VAN STEIRTEGHEM (Bruxelles, Belgique) a montré que si la fonction acrosomique était importante dans le SUZI, elle n'intervenait en aucune manière dans les résultats de l'ICSI. C'est ainsi qu'il a pu obtenir des fécondations et des grossesses dans des cas de globozoospermie.

JeAn Parinaud (Toulouse, France) a rapporté les résultats d'une technique de sélection des spermatozoïdes ayant fait la réaction acrosomique à l'aide d'immunobilles recouvertes par un anticorps dirigé contre la membrane interne de l'acrosome. Cette méthode permet d'obtenir une population contenant près de $90 \%$ de spermatozoïdes réactés et constitue un nouvel outil dans l'étude des caractéristiques de la sous-population de spermatozoïdes aptes à féconder et des événements métaboliques succédant à la réaction acrosomique.

L'acrosome jouant un rôle essentiel dans les processus de la fécondation, il constitue donc une excellente cible pour une contraception. JOHN HERR (Charlottesville, USA) a isolé et cloné une protéine SP10 située dans la matrice et les membranes de l'acrosome. Injectée à des primates, SP10 entraîne la formation d'anticorps qui inhibent la fécondation in vitro chez les bovins. Ces travaux constituent une première approche d'une contraception vaccinale utilisant des antigènes acrosomiques.

En conclusion R.A.P HARRISON a souligné que si de grands progrès dans la compréhension des mécanismes de la capacitation et de la réaction acrosomique avaient été réalisés ces dernières années grâce aux modèles in vitro, il était encore difficile de préciser quels mécanismes étaient privilégiés in vivo. 
Compte-rendu du 15ème congrès mondial sur la fertilité et la stérilité (IFFS)

17/22 septembre 1995, Montpellier

E.SZERMAN-JOLY

Laboratoire de Biochimie A, CHRU Côte de Nacre, 14033 CAEN Cedex

Ce congrès a réuni 3700 personnes de 81 nations différentes. Une partie des communications se rapportait spécifiquement aux problèmes d'infertilité masculine. Des grands thèmes ont été débatus dont voici un aperçu.

\section{EXPLORATION BIOLOGIQUE DE L'HOMME INFERTILE}

- Evaluation de la concentration spermatique (A. ComHaire Belgique).

- Les aspects biochimiques de l'analyse du sperme (W.H. SchILL Allemagne).

- Test de fécondance (A. VeIga Espagne).

\section{APPROCHE RATIONNELLE DE L'IN- FERTILITÉ MASCULINE}

Quelles techniques de microfertilisation : FIV/ICSI/HIC ?

(S. Gorsts Belgique -Hamberger Suède)

Déclin de la fertilité du male

(B. JÉGOU France)

\section{EXPLORATION BIOLOGIQUE DE L'HOMME INFERTILE}

\section{- Evaluation de la concentration sper- matique}

Comhaire a énoncé quelques recommandations pour compter les spermatozoïdes afin d'éviter les erreurs dues à la non homogénéité du sperme et à sa viscosité.
Numération à l'hémocytomètre ;

Il est nécessaire d'adapter la dilution à la concentration spermatique observée après dépôt d'une aliquote entre lame et lamelle. Celle-ci varie de $1 / 10$ è à $1 / 50$ è en fonction de la richesse de départ. La prise d'essai ne doit pas être inférieure à $50 \mathrm{ml}$. Par convention on ne comptera sur la grille que les spermatozoïdes à cheval sur les lignes extérieures sur la partie supérieure et gauche.

On effectuera toujours une deuxième numération avec une seconde aliquote à la même dilution. La variation entre les deux ne doit pas excéder $5 \%$.

Numération dans une chambre de mesure;

La chambre doit être remplie avec $5 \mathrm{ml}$ de sperme non dilué qui pénètre par capillarité ( $3 \mathrm{ml}$ étant un volume trop faible).

Une fois l'appareil calibré, la numération est reproductible et on trouve une bonne corrélation entre les deux techniques.

Comhaire voit un avantage à effectuer la numération sur appareil automatisé par le gain de temps qu'il procure et par la possibilité d'analyser simultanément la mobilité.

\section{- Les aspects biochimiques de l'analyse du sperme}

Schill a présenté un bref aperçu des différents marqueurs biochimiques du sperme pouvant être effectués dans les bilans d'infertilité pour définir trois types d'information.

1. Evaluer le pouvoir fécondant des spermatozoïdes.

2. Déterminer les inflammations chroniques de l'appareil reproducteur masculin,

3. Connaitre les anomalies anatomiques et fonctionnelles

I. Trois marqueurs sont cités pour évaluer la fécondance : acrosine, coloration au bleu d'aniline, ROS (Formation d'espèces radicalaires de l'oxygène) 
L'altération de la fonction acrosomiale est considérée comme un paramètre diagnostic permettant d'évaluer la fécondance du sperme.

L'acrosine est un marqueur intéressant impliqué à la fois dans la liaison du spermatozoïde à la zone pellucide, dans la pénétration, dans la capacitation et la réaction acrosomique. Schill utilise une méthode très simple pour déterminer l'activité protéolytique des spermatozoïdes sur plaque de gélatine. La mesure d'un halo de diamètre compris entre 15 et $25 \mu \mathrm{m}$ autour de la tête du spermatozoïde semble être un bon pronostic de fécondance. Cette technique présente une bonne corrélation avec les dosages biochimiques.

La coloration au bleu d'aniline permet d'évaluer l'immaturité nucléaire. Les histones sont remplacées par les protamines pendant la maturation du spermatozoïde. La persistance des histones est associée à la coloration positive au bleu d'aniline. Un taux supérieur à $25 \%$ indique une immaturité nucléaire.

II. La formation de ROS endommage la membrane spermatique riche en acides gras polyinsaturés entraînant une altération du pouvoir fécondant. La présence de ROS est également corrélée avec une inflammation et avec la présence de leucocytes dans l'éjaculat.

Le dosage de PMN élastase par méthode immunoenzymatique permet également de diagnostiquer une inflammation.

\section{PMN Elastase}

Diagnostic

$<250 \mathrm{ng} / \mathrm{ml}$

Pas d'inflammation

$250-1000 \mathrm{ng} / \mathrm{ml}$

Inflammation modérée

$>1000 \mathrm{ng} / \mathrm{ml}$

Sévère inflammation

D'autres marqueurs tels que le composant C'3 du complément, la ceruléoplasmine ou la présence d' IgG ou d'IgA dans le liquide séminal peuvent également donner des renseignements sur le caractère inflammatoire de l'appareil reproducteur en absence de leucocytes.

III. Parmi les marqueurs des glandes annexes, un taux de fructose abaissé est le reflet de l'atrophie post-inflammatoire des vésicules séminales. Dans le cas d'azoospermie cela indique également une agénésie des vésicules séminales.

L'alpha-glucosidase tend à remplacer actuellement le dosage de carnitine pour différencier une azoospermie sécrétoire d'une azoospermie excrétoire.

Plusieurs marqueurs sont utilisés pour évaluer les sécrétions de la prostate. Schill a cité le Zinc, l'acide citrique, les phosphatases acide prostatiques.

L'intérêt des marqueurs biochimiques dans l'exploration d'une infertilité masculine est d'apporter des informations standardisées ce qui n'est pas le cas des analyses biologiques.

\section{- Test de fécondance}

Veiga a exposé les différents tests disponibles pour évaluer la fertilité masculine. La qualité du sperme doit être analysée en fonction des valeurs standards déterminées par l'OMS qui sont :

volume $>2 \mathrm{ml}$

$\mathrm{pH}: 7,2-8$

concentration $\geq 20.10^{6}$ spermatozoïdes $/ \mathrm{ml}$

$\geq 40.10^{6}$ spermatozoìdes/ éjaculat

mobilité $>25 \%$ mobilité rapide

$>50 \%$ mobilité progressive

vitalité $>75 \%$ spermatozoïdes vivants morphologie $>30 \%$ spermatozoïdes normaux.

Il existe actuellement un certain nombre de tests capables d'apprécier le pouvoir fécondant du sperme, tels que : 
* le Hamster test (fusion avec la membrane de l'ovocyte de hamster)

* hemizona assay (test de fixation à la zone pellucide)

* hypo-osmotic swelling test

* recherche d'anticorps antispermatozoïdes par la technique des immunobilles

* test de survie des spermatozoïdes

* réaction acrosomique

* test de pénétration croisée (étude de l'interaction sperme - mucus cervical)

* analyse biochimique

* microscopie électronique

* analyse chromosomique

En conclusion, chaque laboratoire se doit d'offrir le maximum de techniques permettant d'évaluer le pouvoir fécondant du sperme.

\section{APPROCHE RATIONNELLE DE L'INFERTILITÉ MASCULINE QUELLES TECHNIQUES DE MICRO- FERTILISATION:FIV/ICSI/HIC ?}

Gordts a rappelé l'importance du bilan spermiologique de base devant toute infertilité masculine.

Il comporte :

- dosages hormonaux

- recherche d'anticorps antispermatozoïdes

- réaction acrosomique

- hemizona assay

- choix des différentes techniques de préparation du sperme

- dosage des sites de liaison du manose sur les spermatozoïdes (comme marqueur de la capacité de liaison du spermatozoïde à la zone pellucide).

Il semble généralement admis par de nom- breux auteurs que la préparation des spermatozoïdes en gradient de Percoll donne de meilleurs résultats dans les spermes "pauvres " comparée à la migration ascendante. Selon Aitken l'usage du Percoll protège de la formation de radicaux libres.

Une réaction acrosomique (RA) prématurée est un mauvais pronostic de fécondance. En conséquence, l'évaluation de la RA peut être un facteur nécessaire à la détermination de la technique de préparation du sperme lors de la PMA.

Dans le cas d'infertilité masculine modérée, il n'est pas toujours évident de choisir entre injection intra-cytoplasmique (ICSI) et insémination avec une concentration importante de spermatozoïdes (HIC). L'insémination de $10^{6}$ spermatozoïdes / $\mathrm{ml} /$ ovocyte compense le faible pouvoir fécondant du sperme.

Gordts compare les taux de fécondation lors d'une étude randomisée sur des ovocytes soit microinjectés, soit cultivés en microgouttes avec HIC.Il rapporte également les résultats de deux autres équipes.

\section{TAUX DE FECONDATION}

\section{ICSI HIC}

\section{Hall 95}

$59 \%$

$67 \%$

Tucker 95

$19 \%$

$62 \%$

Gordts 95

$57 \%$

$32 \%$

Il vante les mérites de l'injection intracytoplasmique dans le cas d'azoospermie excrétoire.

Il conclut en mettant en garde contre les problèmes de contamination par erreur technologique pouvant arriver en injectant un DNA étranger dans l'ovocyte en utilisant du polyvinylpyrolidone. L'incidence des anomalies chromosomiques est beaucoup plus élevée chez les patients présentant une oligoasthénotératospermie comparés aux donneurs fertiles. Ces mutations ou anomalies chromosomiques peuvent être cause de la stérilité et pourront être transmises à la 
descendance si une microinjection est effectuée.

Hamberger a fait un remarquable exposé dans lequel il dénonce le développement immodéré des techniques de micromanipulation en rappelant les risques divers non encore déterminés dont l'utilisation de substances "toxiques".

Le but de sa présentation consiste à limiter l'injection intra-cytoplasmique (ICSI) aux indications qui la nécessitent absolument quand une FIV classique ne peut être effectuée ou a de très faibles chances de réussite.

Après avoir rappelé l'importance de la femme sur la qualité du conceptus, il a cité de meilleurs résultats obtenus en ICSI dans les indications masculines ( $23 \%$ de grossesses par cycle) par rapport aux échecs de FIV (14\% de grossesses par cycle).

A la question : "Doit-on passer en ICSI après un échec de FIV classique? ", il donne les résultats de son équipe qui, pendant deux ans, a choisi après un échec de FIV (ou un taux de segmentation inférieur à 15 $\%)$ de comparer lors d'un second cycle FIV et ICSI sur un rapport 50/50 des ovocytes.

Pour un tiers des cas un nouvel échec en FIV se produit avec des embryons en ICSI et pour $2 / 3$ des cas des embryons sont obtenus en FIV (taux de segmentation $50 \%$ ) et en ICSI (taux de segmentation $70 \%$ ), ce qui prouve que pour la majorité des patients il n'est pas nécessaire de passer en ICSI dès la deuxième tentative.

La présence d'autoanticorps dans le sperme est également souvent une indication d'ICSI de première intention. Or Hamberger et coll. rapportent de bons taux de succès en FIV classique. Leur pratique consiste à effectuer lors d'un premier cycle une comparaison IVF/ICSI (rapport 50/50 des ovocytes). Si un bon taux de segmentation est obtenu en FIV classique, l'ICSI ne sera plus utilisée dans le cycle suivant.
En ce qui concerne la tératospermie, l'ICSI est une bonne indication quand le taux de spermatozoïdes normaux est inférieur à $5 \%$ (Classification de Krugger). Mais, si le taux de spermatozoïdes normaux est supérieur à $5 \%$, ICSI et HIC (High Insemination Concentration) donnent des résultats comparables.

Dans les spermes " pauvres", le test de sélection des spermatozoïdes est un élément de décision quant à la technique à utiliser.
Test de Sélection

$>10^{6}$ spermatozoïdes mobiles

$0,210^{6}$ à $10^{6}$

spermatozoïdes mobiles

$<0,210^{6}$ spermatozoïdes mobiles
Technique

IVF

IVF ou ICSI

ICSI
L'ICSI reste la technique exclusive dans les indications d'aspiration de spermatozoïdes épididymaires (MESA) ou extraction de spermatozoïdes testiculaires (TESE).Cette technique est également recommandée en association avec le diagnostic préimplantatoire.

Dans un bon nombre d'indications il semble judicieux d'optimaliser les conditions de FIV avant d'envisager une ICSI.Par exemple, lors du recueil de sperme on peut utiliser des antioxydants pour éviter les peroxydations membranaires qui augmentent le taux de fragmentation des embryons. Les microgouttes ou HIC sont également des techniques efficaces pour la majorité des couples infertiles.

En conclusion un échec de FIV classique n'est pas une indication d'ICSI. 
DÉCLIN DE LA FERTILITÉ DU MÂLE

Récemment une analyse de 61 communications publiées dans le monde a mis l'accent sur une diminution du nombre de spermatozoïdes entre 1938 et 1990 . Cette étude intéressante est très controversée ; les populations étudiées et la méthodologie de l'évaluation du sperme étant variables dans les différentes publications. Son mérite est d'avoir suscité l'intérêt de nombreuses équipes pour étudier le déclin de la spermatogenèse.

B. Jégou a présenté les résultats de certaines de ces équipes : B. Auger (Bicêtre) et al. prétend qu'à Paris la concentration en spermatozoïdes chez les hommes fertiles (donneurs de sperme) n'a cessé de décroître ces 20 dernières années $\left(89 \times 10^{6} / \mathrm{ml}\right.$ en 1973 vs $60 \times 10^{6} / \mathrm{ml}$ en 1992 ) ainsi que la qualité du sperme. Les analyses ont été faites dans le même laboratoire sans modification des techniques.

D'autres équipes en Ecosse, Belgique et Angleterre rapportent les mêmes déclins. Par contre, pendant la même période, aucune altération n'a été trouvée à Oslo, Toulouse ou Munster suggérant que des variations géographiques peuvent exister.

\section{Quelles hypothèses peut-on évoquer pour expliquer ces altérations?}

On remarque pendant cette période une augmentation des anomalies de l'appareil urogénital, de la fréquence des cancers tes- ticulaires ainsi que du taux de cryptorchidie ou de cancer de la prostate chez l'homme. Des anomalies comparables ont été observées chez les animaux (panthères en Amérique du Nord, alligators en Floride, oiseaux, poissons, reptiles), ce qui permet d'évoquer le rôle négatif des facteurs de l'environnement.

B. Jégou décrit quelques arguments en faveur de cette hypothèse :

L'homme vit dans une mer d'oestrogènes (pollution hormonale) ou oestrogènes "like " utilisés essentiellement dans l'agriculture. Des anomalies génito-urinaires et spermatiques ont été décrites pour des souris, panthères et probablement des hommes exposés aux oestrogènes in utero. La vulnérabilité du testicule à ces agents physiques et chimiques peut expliquer l'origine de la tendance à la diminution.

Il est évident qu'il n'existe pas d'incidence directe entre concentration spermatique et fertilité mais on peut supposer qu'une chute de 20 à $50 \%$ de la numération spermatique entraînera une augmentation de la population d'hommes hypofertiles nécessitant une assistance médicale à la procréation avec tout ce qui l'accompagne comme augmentation des dépenses budgétaires (problème économique et social).

B. Jégou conclut en invitant l'assemblée à participer aux recherches dans ce domaine et en présentant l'étude multicentrique Française PERE (Perturbation \& Environmental Research). 


\section{DIFFÉRENTS TYPES D'ALTÉRA- TIONS DE LA SPERMATOGÉNÈSE PROVOQUÉS PAR DES DÉLÉTIONS DU CHROMOSOME Y EMPORTANT LE GÈNE D'UNE NOUVELLE PROTÉI- NE DE LIAISON DE L'ARN.}

R ReiJo, TY LeE, P Salo, R Alagappan, LG Brown, M Rosenberg, S Rozen, T JaFfe, D Straus, O HovatTa, A Delachapelle, S SILBER, DC PAGE.

Howard Hughes Medical Institute and Center for Genorne Research Whitehead Institute and Department of Biology, Massachusetts Institute of Technology, Cambridge, MA, USA \& Department of Medical Genetics, University of Helsinki, Helsinki, Finland \& Department of Biology, Brandeis University, Waltham, MA, USA \& Family Federation of Finland, Helsinki, Finland \& In Vitro Fertilization Program, St Luke's Hospital, St.Louis, MO, USA.

Nature Genetics. 1995;10:383-393.

En utilisant 84 sondes nucléotidiques se localisant tout au long du chromosome $\mathrm{Y}$, des microdélétions ("micro" = non visibles au caryotype) ont été mises en évidence au niveau du bras long du chromosome Y pour $13,5 \%$ (12/89) des cas d'azoospermie non obstructive étudiés. Aucune microdélétion du bras long du chromosome $\mathrm{Y}$ n'a été retrouvée chez les pères et autres membres de la famille des sujets, ni chez 90 sujets fertiles. Ces délétions sont supposées survenir de novo. Les 12 délétions se recouvrent et définissent une région contenant un ou plusieurs gènes nécessaire(s) à la spermatogénèse et correspondant à ce qui a été nommé AZF (azoospermic factor). La délétion de la région AZF s'associe à des altérations variables de la spermatogénèse chez les 9 patients ayant eu une biopsie, depuis une absence complète de cellules germinales (Sertoli-cell-only syndrome ; 5 cas) jusqu'à des blocages de la spermatogénèse ( 4 cas) avec d'occasionnelles évolutions jusqu'au stade de spermatides à noyau condensé ( 2 cas).

L'étude détaillée du recouvrement des ces délétions montre qu'elles intéressent toutes une région de 500 kilobases. En examinant plus en détail cette région, les auteurs ont trouvé une unité de transcription codant pour une protéine de 366 acides aminés dont 85 représentent une séquence consensus RNP/RRM, observée dans de nombreuses protéines liant l'ARN ou l'ADN simple brin. Ce gène appelé DAZ (Deleted in AZoospermia) est transcrit spécifiquement dans le testicule adulte (ARN messager de $3,5 \mathrm{~kb}$ ).

Cette protéine DAZ représente un candidat pour l'AZF. Des investigations complémentaires sont nécessaires pour préciser son rôle dans la spermatogénèse.

\section{Commentaire (Hervé LEJEUNE)}

L'avancée des recherches portant sur l'origine génétique de certains troubles de la spermatogénèse est rendue difficile du fait même que l'expression phénotypique du trait pathologique empêche sa transmission. Or, c'est souvent le caractère transmissible d'une affection qui oriente vers une origine génétique. Les anomalies cytogénétiques représentent par contre une cause connue de longue date de déficit de la spermatogénèse. C'est à partir de la constatation de délétions de l'extrémité du chromosome $Y$, visibles au caryotype, qu'est né le concept de l'azoospermic factor, $A Z F$. Cet article rapporte lisolement d'un gène ayant apparemment toutes les qualités pour représenter l'AZF.

La fréquence $(13,5 \%)$ des microdélétions de la région de l'AZF survenant de novo dans les azoospermies non obstructives est notoirement élevée. Il semble s'agir d'une zone du génome particulièrement vulnérable, soumise à des remaniements fréquents, ce qui peut s'expliquer par l'existence, dans le chromosome $Y$, de zones répétitives, qui exposent à des erreurs fréquentes lors des crossing over. Ces délétions de novo s'expriment d'emblée 
puisque les gènes de cette région du chromosome $Y$ sont présents en 1 seul exemplaire.

Non moins notoire est la variabilité dans l'expression phénotypique au niveau de l'histologie testiculaire. On aurait pu s'attendre à ce que des anomalies génétiques apparemment très proches, comme dans les cas étudiés ici, donnent des anomalies histologiques homogènes. Or, syndrome des cellules de Sertoli isolées et blocage de la spermatogénèse avec évolution jusqu'à des formes condensées dans quelques rares tubules paraissent représenter différents aspects du déficit d'un seul gène. Ceci semble souligner que le gène $D A Z$ n'est que l'un des nombreux gènes intervenant dans la spermatogénèse. Sa suppléance, en cas de délétion, peut être partiellement assurée chez certains des sujets et dans certains de leurs tubes séminiferres.

Les gènes appelés YRRM (Y chromosome $R N A$ recognition motif) précédemment décrits comme candidats potentiels pour $A Z F$ se révèlent être présents chez les sujets, présentant une délétion dans la région $A Z F$, étudiés ici. Il apparaît toutefois que ces gènes ont des homologies de séquence avec $D A Z$, mais en sont distincts; ils se situent, en plusieurs exemplaires, dans d'autres régions du chromosome Y. Les protéines codées par les gènes DAZ et YRRM ont en commun une séquence de liaison à l'ARN. Une situation homologue a été décrite chez la drosophile, la délétion d'un gène codant pour une protéine liant l'ARN provoque une perte de cellules germinales. Des protéines liant l'ARN et ainsi potentiellement impliquées dans des modifications post transcriptionnelles, paraissent importantes pour la spermatogénèse.

L'identification du gène $D A Z$ va permettre des études complémentaires certainement importantes dans la compréhension de la physiologie de la spermatogénèse, et permettra de rechercher d'autres anomalies de ce gène comme des mutations ponctuelles.
OSCILLATIONS DU CALCIUM DANS

LES GUFS DE MAMMIFÈRES

DÉCLENCHÉE PAR UNE PROTÉINE SOLUBLE DES SPERMATOZOÏDES.

J.PARRINGTON, K.SWANN, v.I.SCHECHENKo, A.K.SESAY, F.A.LAL

MRC National Institute for Medical Research, Mill Hill, London MRC Experimental Embryolo-

gy and Teratology, St Georges Hosp.London.

Departement of Anatomy and Dev..Biology,

Univ.College, Gower Street, London.

Nature, V.379, 25-01-1995, 364-368.

Chez les mammifères, au moment de la fécondation le spermatozoïde induit dans l'ovocyte une série d'oscillations de $\mathrm{Ca}^{2+}$ qui sont les principaux déclencheurs de l'activation de l'ovocyte et du développement précoce de l'embryon. Cependant, jusqu'à présent, on ne savait pas par quel mécanisme le spermatozoïde déclenchait le phénomène initial.

Les extraits de spermatozoïdes, qui entraînent des oscillations calciques identiques à celle que l'on observe au cours de la fécondation, correspondent à des mélanges complexes de protéines. Nous avons isolé par chromatographies des protéines solubles extraites de spermatozoïdes de hamster et testé le pouvoir " oscillogène" des diverses fractions sur des ovocytes de souris. L'oscillation calcique est mise en évidence par un colorant fluorescent sensible au $\mathrm{Ca}^{2+}$. L'activité oscillogène paraît appartenir à une protéine de masse moléculaire $33.000 \mathrm{dont}$ nous avons pu établir la structure primaire.

Les résultats de l'étude immunocytochimique montrent que la protéine est localisée dans un site intracellulaire de la région du segment équatorial du spermatozoïde. Nous suggérons d'appeler cette molécule oscilline.

Quelques observations que nous n'avons pas encore publiées nous font supposer que certaines stérilités masculines pourraient être dues à l'absence d'oscilline. 
L'injection d'extraits de spermatozoïdes provoque des oscillations calciques dans des cellules comme les neurones et les hépatocytes. Il est donc possible que l'oscilline puisse être impliquée dans les décharges de $\mathrm{Ca}^{2+}$ des cellules somatiques.

Commentaire (J.C.CZYBA)

Ce très beau résultat apporte beaucoup à la connaissance des mécanismes de la fécondation.

Cependant, malgré la prudence des auteurs, on apprend, une fois de plus, à la lecture de la presse de vulgarisation scientifique que la cause de la stérilité masculine vient d'être découverte.

\section{ENDOCRINOLOGIE}

ANALYSE QUANTITATIVE DU MESSAGER DU RECEPTEUR DES ANDRO. GENES DANS LES CELLULES DE LEYDIG ET LES CELLULES DE SERTOLI EN COURS DE DEVELOPPEMENT PAR HYBRIDATION IN SITU Li-XIN SHAN, LI-JI-ZHU, C.W.BARDIN ET
M.P.HARDY

Endocrinology 136, 9, 3856-3862, 1995

La testostérone produite par les cellules de Leydig est nécessaire pour le maintien de la spermatogenèse par les cellules de Sertoli chez le rat. Cependant, la présence du récepteur (RA) dans les cellules de Leydig du rat prépubère, suggère un rôle des androgènes dans la fonction des cellules de Leydig jeunes et au cours de la différenciation.Dans cette étude, la distribution du mRNA de RA a été examinée au 21ème, 35 ème et 90 ème jour post-partum par hibridation in situ non radioactive.

Le marquage des cellules de Leydig a été observé à tous les âges étudiés mais son intensité varie au cours du développement. Elle est intermédiaire au niveau des cellules de Leydig jeunes (à 21 jours), maximale à 35 jours et la plus basse à 90 jours correspondant aux cellules de Leydig adultes.

Un marquage des cellules de Sertoli à tous les âges a été observé mais l'intensité est différente de celle des cellules de Leydig. L'intensité de la coloration varie avec le stade de la spermatogenèse; ce marquage devient notable au stade IV, atteint son plus haut niveau aux stades VII - VIII et devient pratiquement nul aux stades IX à XI. Par ailleurs, il est connu que les cellules péritubulaires et les péricytes contiennent 
du récepteur des androgènes. En ce qui concerne les cellules germinales, la situation est beaucoup plus floue. Il est probable que soit l'action de la testostérone s'exerce par l'intermédiaire de la cellule de Sertoli, soit la testostérone est transportée par l'ABP ou les deux mécanismes à la fois. (M.DROSDOWSKY)

\section{ETUDE PROSPECTIVE DES PROFILS HORMONAUX ET SÉMINAUX CHEZ DES COUREURS DE MARATHON}

\section{C.E.JENSEN, K.WISWEDEL, J.MC.LOUGHLIN, Z.VAN DER SPUY}

Depart. of Obstetrics and Gynaecology, University of Cape Town, Groote Schuur Hospital, Cape Town, Afrique du Sud..

Fertility and Sterility, déc. 1995, V.64, n 6 , 1189-1196

La course de marathon est devenue une activité sportive et récréative très répandue. Un retentissement sur la physiologie endocrinienne a déjà été signalé en particulier chez les femmes. Peu d'études ont été publiées quant aux effets du sport sur la spermatogenèse.

Nous avons conduit une étude d'une année chez 24 volontaires coureurs de marathon âgés de 25 à 54 ans. Les coureurs ont été répartis chaque mois en deux groupes : entraînement léger (moins de $55 \mathrm{~km} / \mathrm{semai}$ ne) et entraînement lourd (entre 60 et $160 \mathrm{~km} /$ semaine). Le marathon $(56 \mathrm{~km})$ a été couru 5 mois après le début de l'étude. Durant les 7 derniers mois, l'entraînement s'est poursuivi, diminuant de $60 \mathrm{~km}$ à
$40 \mathrm{~km} / \mathrm{sem}$.

Les dosages hormonaux (LH, FSH,T,P, PRL, E2) et les spermogrammes ont été effectués 8 fois (de décembre à novembre).

\section{Résultats}

L'augmentation progressive de l'entraînement pendant les 5 premiers mois s'est accompagnée de façon significative d'une augmentation des taux sériques de PRL et d'une diminution des taux de progestérone. Nous n'avons pas noté de variations significatives des autres hormones.

Le volume du sperme et la mobilité sont significativement diminués le mois du marathon. La numération n'est pas modifiée. La tératospermie augmente pendant toute l'année de l'étude.

\section{Discussion}

Les variations hormonales que nous avons constatées sont difficilement comparables à celles relevées par d'autres auteurs dans des circonstances différentes, dans des durées plus courtes. Les variations du spermogramme ne paraissent pas susceptibles de diminuer la fertilité, sauf peut être l'augmentation de la tératospermie.

\section{Commentaire (J.C.CZYBA)}

Cet article apporte des données nouvelles mais peu interprétables. Que signifie l'aug. mentation de prolactine et la diminution de progestérone? On peut s'étonner de l'absence de modification des taux de gonadotropines et de testostérone.

Les modifications du spermogramme posent effectivement le problème d'un retentissement éventuel sur la fertilité. Il n'y a pas de réponse ici, le débat reste ouvert. 


\section{IMMUNOLOGIE}

\section{MECANISME DES REJETS DE GREFFES}

\author{
DaVID L. VAUX \\ Institut de recherche médicale Walter et Elisa \\ Hall, Hopital Royal Melbourne, Australie.
}

Nature, 1995, $377: 576$ - 577.

A l'inverse des autres tissus, les greffes testiculaires ne sont pas rejetées lorsqu'elles sont transplantées à des sujets non compatibles dans le système H.L.A. Bellegrau et son équipe ont récemment montré que les greffes testiculaires entre souris non compatibles dans le système A.B.C. (équivalent du système H.L.A. chez la souris) n'étaient pas rejetées.

Les cellules testiculaires exprimaient à leurs surfaces le ligand de C.D. 95. Inversement, les greffes de testicules de donneurs n'exprimant pas le ligand de CD 95 étaient rapidement rejetées.

Le tissu testiculaire est un nouveau champ d'expérimentation dans la compréhension des mécanismes de rejet de greffes. Dans le futur, les transplants exprimant un ligand de C.D. 95 transgénique pourraient être utilisés.

L'objectif principal du système immunitaire est la défense de l'organisme contre les agressions extérieures. Toute pénétration dans l'organisme d'allo-antigène entraîne une activation des lymphocytes T et $\mathrm{B}$. La réaction immunitaire devient un handicap dans des situations bien particulières comme la greffe d'organes. Trois organes sont des sites immunitaires "priviligiés" : le testicule, la chambre antérieure de l'oeil, le cerveau. Les allo et xénogreffes ne sont pas rejetées. Si pour l'oeil et le cerveau les mécanismes sont relativement simples (ces organes sont dépourvus de circulation lymphatique), il n'en est pas de même pour le testicule qui, lui, possède un réseau de vascularisation lymphatique important.

Un des mécanismes de rejet de greffe passe par les lymphocytes T activés. Ces derniers reconnaissent l'allo-antigène exprimé à la surface membranaire. Lors de l'activation , les lymphocytes T expriment C.D. 95, qui lorsqu'il est mis en contact avec son ligand, délivre un signal d'apoptose aux lymphocytes T eux mêmes. C.D. 95 serait un des mécanismes d'arrêt de la réponse immunitaire.

Les souris transgéniques qui expriment un gène muté pour C.D. 95 ou le ligand de C.D.95 développent des pathologies autoimmunes résultant de la diminution de l'apoptose lymphocytaire. Le ligand de C.D. 95 est exprimé en grande quantité dans le testicule. Lors d'une allogreffe testiculaire non compatible, la réaction immunitaire se déroulerait normalement. Seuls les lymphocytes $\mathrm{T}$ activés, spécifiques de l'antigène et exprimant C.D. 95, sont rapidement détruits par le ligand des cellules testiculaires.

Ces découvertes ont des implications directes dans la transplantation. Les lymphocytes $\mathrm{T}$ et $\mathrm{B}$ sont les principaux acteurs des rejets d'allogreffes et bien sûr, a fortiori, des xénogreffes. Greffer des organes transgéniques exprimant le ligand de C.D. 95 pourrait être une voie de recherche interessante. Chez ces patients, les lymphocytes $\mathrm{T}$ activés, spécifiques d'antigènes du greffon, seraient détruits par la greffe elle-même. Le reste du système immunitaire ne serait pas affecté et resterait intact.

Il y a bien sûr loin de la théorie à la pratique. La mise en jeu du système cellulaire n'est qu'un des aspects du rejet de greffe. Les anticorps circulants et l'activation de la cascade du système du complément sont 
aussi un des mécanismes principaux des rejets aigüs. C'est tout de même une voie de recherche interessante.

\section{Commentaires (B. SALLE)}

Le travail de Bellgrau commenté par Vaux explique le mécanisme de tolérance immunitaire des greffes testiculaires. Brinster dans ses expérimentations ne décrit pas le mécanisme d'acceptation des greffes. Le ligand de CD 95 n'est surement pas le seul antigène mis en jeu dans la tolérance immunitaire. Dans le testicule la barrière Hémo testiculaire peut, peut être, empécher les autres acteurs du rejet de greffes d'entrer en action.

Les champs d'applications de cette découverte dépassent largement le domaine de l'andrologie. La compréhension du rejet de greffes permettra de mieux adapter les outils thérapeutiques et de se diriger vers la xenogreffes à l'aide d'animaux transgéniques.

\section{CANCER}

\section{CANCER ET STERILITE MASCULINE}

\author{
D.MeIROW, J.G.SchENKER
}

Human Reproduction 10 (8) : 2017-2022, 1995

Il s'agit d'un article de revue de la littérature récente, issu d'un service de gynécologie obstétrique. Il est formé de 3 grands chapitres :

A. Une revue de l'impact des cancers en général sur la fertilité. L'hypercatabolisme accompagnant les pathologies tumorales peut être la cause d'une freination hypothalamique. Les cancers du testicule sont classiquement associés à des troubles de la fertilité, $60 \%$ des patients présentant une oligospermie nette. Trois mécanismes peuvent être en cause : des troubles endocriniens, comme l'hypersécrétion de $\beta$ HCG par la tumeur, un mécanisme immunitaire (dont il faut signaler que l'importance du taux d'anticorps est proportionnelle au stade tumoral), et enfin des mécanismes intratesticulaires locaux soit par fibrose testiculaire, soit par carcinome in-situ (dont l'impact actuel est actuellement revu à la baisse dans la littérature urologique), ou même par des testicules ne comprenant plus que des cellules de Sertoli. Enfin la chaleur locale secondaire à l'hypervascularisation tumorale pourrait aussi parfois être en cause. Les cancers endocriniens purs sont exceptionnels, comme les tumeurs de la région hypothalamique ou des surrénales. Enfin les homéopathies, et particulièrement la maladie de Hodgkin peuvent être impliquées. Dans cette dernière, la fertilité est abaissée chez $70 \%$ des hommes malades, alors que par ailleurs le bilan endocrinien est normal. Ces anomalies de la fertilité 
sont indépendantes du stade tumoral, ne sont pas dues à un effet tumoral local, ou à un envahissement testiculaire, mais seraient principalement d'origine immunitaire.

B. L'impact des traitements sur la fertilité est revu. Il varie selon les modalités utilisées (chirurgie, chimiothérapie, radiothérapie, association). Le problème principal est alors de limiter au maximum les séquelles à long terme. En ce qui concerne la radiothérapie, il existe une oligospermie transitoire vers $0,1 \mathrm{~Gy}$ (dose d'un rayonnement diffusé), et une azoospermie irréversible dès 2 Gy. C'est dire l'importance de la protection gonadique. En ce qui concerne la chimiothérapie, les séquelles varient selon les drogues utilisées, leurs doses, les associations effectuées. En particulier si le protocole BEP (utilisé dans les cancers testiculaires) présente une assez bonne réversibilité, le MOPP (utilisé dans les maladies de Hodgkin) donne lui une stérilité irréversible.

C. La protection de la fertilité repose sur les mesures de radioprotection et sur le choix des drogues les moins délétères pour la spermatogénèse. Mais il repose surtout sur la mise systématique du sperme en banque avant tout traitement. Une limitation ancienne existait, quant à la possibilité pour le sperme de tenir la congélation ( $23 \%$ dans les Hodgkin, $50 \%$ dans les cancers testiculaires). Celle-ci pourrait être tournée grâce aux progrès de la procréation médicalement assistée (ils évoquent la SUZI) et doivent conduire à ne plus refuser de congélation sous prétexte de mauvaise qualité en décongélation. Enfin il n'existe pas de protection hormonale ayant fait la preuve de son efficacité chez l'homme. Quant aux effets génétiques sur la descendance, les auteurs terminent en signalant que si les données actuelles permettent d'être assez rassurant, elles ne permettent pas de conclure définitivement.

\section{Commentaires (N. MOTTET) :}

Il s'agit d'un article intéressant, facile à lire, qui fait un point actuel sur cette question rarement traitée. Elle trouve pourtant toute son importance dans cette population d'hommes jeunes dont la guérison est de plus en plus fréquente grâce aux progrès de la chimiothérapie, et dont l'interrogation principale devient maintenant celle des séquelles à long terme des traitements. Les 55 références sont récentes. On peut seulement regretter que les auteurs ne rappellent pas la nécessité d'une contraception efficace pendant l'année qui suit la fin du traitement, les anomalies génétiques étant maximales lors de la période de recolonisation testiculaire.

La lecture de cet article est très recommandable à tous ceux qui s'intéressent à la fertilité, ainsi qu'aux cancérologues. 


\section{FÉCONDATION IN VITRO}

\section{DÉVELOPPEMENT DE SOURIS NOR- MALES À PAR'TIR D'OVOCY'TES INJECTÉS AVEC UN NOYAU DE SPERMATOCYTE SECONDAIRE.}

YASUYUKI KIMURA \& R. YANAGIMACHI

Department of Anatomy \& Reproductive Biololy, University of Hawaii, School of Medecine, Honolulu, HI 96822 USA.

Biology of Reproduction, 1995;53:855-862.

Cette étude, réalisée chez la souris, montre l'obtention d'un développement embryonnaire normal, donnant des souris normales et fertiles, par microinjection d'un noyau de spermatocyte secondaire dans un ovocyte mature. Les cellules spermatogénétiques ont été obtenues à partir de tubes séminifères de souris adultes. Les spermatocytes secondaires ont été sélectionnés selon la taille de la cellule et celle du noyau. L'analyse a posteriori du contenu chromosomique a montré que les cellules sélectionnées étaient effectivement des spermatocytes secondaires dans $86 \%$ des cas. Les noyaux des spermatocytes secondaires ont été isolés du cytoplasme et injectés individuellement dans des ovocytes matures. Ce processus n'activant pas les ovocytes, ceux-ci ont été électroactivés 2 heures après l'injection du noyau, au moment où les chromosomes prématurément condensés se trouvent associés aux microtubules du fuseau. A la suite de l'électroactivation, les chromosomes de l'ovocyte et du spermatocyte secondaire terminent leur seconde division méiotique, avec expulsion de deux globules polaires indépendants et formation d'un pronucleus mâle et d'un pronucleus femelle dans $75 \%$ des cas. Des embryons au stade 2 ou 4 cel- lules provenant de tels ovocytes ont été transférés chez des femelles. $22 \%$ des embryons se sont développés et ont donné des souris normales et fertiles. Les descendants des souris obtenues par cette procédure étaient tous normaux.

Les résultats de cette étude indiquent que l'empreinte gamètique (imprinting) des cellules spermatogénétiques est réalisée chez la souris avant ou pendant la seconde division de méiose.

\section{Commentaire (Hervé LEJEUNE)}

Dans leur travail précédent (voir commentaire dans Andrologie 1994;4:532-533), $R$. Yanagimachi et ses collaborateurs présentaient le développement de souris à partir de spermatides. La condition limite pour obtenir un développement embryonnaire normal semblait alors l'introduction dans l'ovocyte mature d'un génome haploïde. Il apparaît ici que l'introduction dans l'ovocyte mature de 2 génomes haploïdes, il est vrai prêts à se séparer, donne des résultats similaires. On observe l'expulsion d'un des génomes haploïdes mâles sous forme d'un globule polaire comme cela se produit pour l'un des génomes haploüdes femelles. L'ovocyte apparaît ainsi capable de soutenir la deuxième division de méiose mâle, comme il le fait pour son propre compte. La chronologie entre microinjection et électroactivation doit toutefois être précise pour laisser le temps ( 22 heures) aux génomes haploüdes mâles de débuter une condensation en chromosomes et de s'associer aux microtubules fusoriaux.

Ainsi les limites permettant d'obtenir un développement embryonnaire normal reculent encore d'un stade. Pourra-t-on aller encore plus loin? On n'ose plus prétendre que la limite de faisabilité est atteinte. On note que $14 \%$ des ovocytes ont été injectés avec des spermatocytes primaires qui ont été confondus avec des spermatocytes secondaires lors de la sélection sur les critères morphologiques. Après électroactivation on 
constatait dans ces cas, un pronucleus mâle de taille double de celle du pronucleus femelle et une triploïdie (ces zygotes triplö̈des n'ont pas été transférés). Toutefois, si on suit le principe d'utiliser, pour soutenir la méiose mâle, l'ovocyte à un stade synchrone avec le spermatocyte, il faudrait, pour utiliser un spermatocyte $I$, avoir recours à des ovocytes primaires fotaux ou au moins ceux des follicules primordiaux... (à suivre !)

Si les expériences précédentes utilisant les spermatides rondes ont été rapidement appliquées dans l'espèce humaine dans des cas de blocage de la spermiogénèse, le champ d'application potentiel de l'utilisation des spermatocytes secondaires apparaît moins clairement. Les cas de blocage de la spermatogénèse au stade de spermatocytes II ne semblent pas fréquemment reconnus sur les biospies testiculaires. L'idée même de transposition de ces expériences à l'espèce humaine a, par ailleurs, de quoi inquiéter même les plus confiants dans la sélection naturelle qui s'opère au cours du développe- ment embryonnaire. De tels protocoles mettant en jeu aussi directement le génome (séparation mécanique du noyau et du cytoplasme, microinjection du noyau du spermatocyte $I I, 2^{\circ}$ division de méiose in vitro, dans l'ovocyte) impliquent un risque potentiellement accru d'erreurs. Si on soumet le "filtre" de la sélection naturelle (qui est tout de même imparfait) à un nombre accru d'embryons à contenu génétiquement anormal, on risque d'augmenter statistiquement les anomalies génétiques qui passent le filtre du développement embryonnaire et fotal. Comme il est de mise de réaliser des études toxicologiques précises avant mise sur le marché de tout médicament, des études chez l'animal comportant l'analyse des embryons non évolutifs, apparaissent nécessaires pour préciser le risque d'anomalies du génome potentiellement induites par les micromanipulations des gamètes mâles immatures. 